\title{
STATUS OF NEG COATING AT ESRF
}

\author{
M. Hahn, R. Kersevan*, ESRF, Grenoble, France
}

\begin{abstract}
The ESRF non-evaporable getter (NEG) coating facility is in operation since two years now. A large part of the insertion device straight sections of the electron storage ring has been equipped with in-house coated $5 \mathrm{~m}$ long aluminum vacuum chambers with an inner vertical aperture of $8 \mathrm{~mm}$. Operational experience with different coating parameters leading to different film thicknesses will be given and compared to bremsstrahlung data. The paper deals also with improvements of the coating production and chamber preparation, and describes some aspects of NEG coating data acquisition, visualization, and remote control. The R\&D program leading to a more powerful DC solenoidal coating tool to further improve the NEG coating production throughput and quality aspects is also discussed.
\end{abstract}

\section{INTRODUCTION}

The vacuum system of the ESRF storage ring consists of welded stainless steel chambers with brazed copper absorbers with a cross-section of $74 \mathrm{~mm}$ (horizontal) by $33 \mathrm{~mm}$ (vertical) for all chambers inside quadrupole and sextupole magnets [1]. Apart from the dipoles, the intense synchrotron radiation (SR) is produced in a straight section containing a five meter long flat insertion device (ID) vacuum chamber with connection chambers for pumping by means of sputter- ion pumps (SIP), beamposition monitors, bellows and RF contact fingers for compensation of the elongation during in-situ bakeout. The pressure bump over the length of an ID chamber can be considerable which may lead to problems with bremsstrahlung radiation especially when the electron beam collides with residual gas molecules during the initial conditioning phase following the installation.

Starting in 1998, the ESRF has pursued an aggressive program of R\&D, for the characterisation of NEG-coated chambers under SR bombardment [6].

\section{INSERTION DEVICE CHAMBERS}

Table 1 gives some details concerning the NEG-coated ID chambers which are installed in the storage ring as of May 2005. Three NEG-coated chambers out of 316 LN with a section of $57 \mathrm{~mm}$ by $8 \mathrm{~mm}$ and $50 \mathrm{~mm}$ by $8 \mathrm{~mm}$ have already been replaced by the extruded aluminium chambers with comparable beam chamber geometry for machine impedance reasons [5]. With the exception of one, all $57 \mathrm{~mm} \times 8 \mathrm{~mm}$ section aluminium chambers have been NEG coated in the vacuum labs at ESRF. The 2093 $\mathrm{mm}$ long chambers are installed in front of in-vacuum undulators to provide an additional conventional SR source in those straight sections.

*On sabbatical leave at the SNS/ORNL, Oak Ridge, USA
Table 1: List of NEG-coated ID vacuum chambers. Material 1 is extruded-aluminium, 6060. Mat. 2 is $316 \mathrm{LN}$

\begin{tabular}{|c|c|c|}
\hline $\begin{array}{c}\text { Material / } \\
\text { length }(\mathbf{m m})\end{array}$ & $\begin{array}{c}\text { Section }(\mathbf{h} \mathbf{x} \mathbf{v}) \\
\left(\mathbf{m m}^{\mathbf{2}}\right)\end{array}$ & quantity installed \\
\hline $1 / 5073$ & $74 \times 11$ & 2 \\
\hline $1 / 5073$ & $57 \times 8$ & 9 \\
\hline $1 / 2093$ & $74 \times 11$ & 4 \\
\hline $2 / 5175$ & $57 \times 8$ & 1 \\
\hline
\end{tabular}

Since the end of 2004, the ESRF has adopted for its out-of-vacuum ID vacuum chambers a NEG deposition thickness of around $500 \mathrm{~nm}$, while the chambers coated before had received around $1 \mu \mathrm{m}$. As predicted by static vacuum tests [8], the vacuum performance did not suffer, while the coating production duration could be reduced significantly.

\section{QUADRUPOLE CHAMBERS}

The NEG coating is a reliable and cost-efficient solution that provides also advantages during the chamber installation procedure, leading to reduction of downtime of the accelerator. Due to the very low photon-induced desorption rate, SR sources under construction plan to use the NEG coating in addition to lumped pumps in order to gain conditioning time [7].

\section{"Pump-less Design"}

Based on the extensive experience accumulated at the ESRF, and on previous studies [9-11], we postulate that a SR light source vacuum system could be designed and operated where, apart from the dipole and crotch absorber area and the upstream and downstream insertion device chambers, the only source of pumping would be the NEG-coated vacuum chambers. This means that a very limited number of lumped pumps would be necessary, and we therefore call it a "pump-less design". The wallpumping effect would provide the little pumping necessary to keep the partial pressures of non-getterable gases low, mainly methane's. The "SOLEIL-type" chamber described below, has demonstrated that such a solution would be feasible. Another test involving a smaller chamber, another pump-less solution, with more stringent constraints on its specific conductance, is in preparation now at the ESRF and will be discussed further below.

\section{"SOLEIL- type" Cross-Section}

In December 2004 ESRF has replaced a $3530 \mathrm{~mm}$ long vacuum chamber (called CV3) at the entry of one of the standard vacuum sections by a NEG-coated aluminum 
chamber without the lumped pumps (two 200 1/s GP200 NEG pumps by SAES, and one Varian StarCell 45 1/s SIP) which are usually installed on this family of chambers. A piece of aluminium extrusion designed for the quadrupole chambers of the French synchrotron light source SOLEIL (under construction) has been adapted by welding bi-metal Al-Stainless Steel flanges to fit the ESRF storage ring. Two additional cold-cathode gauges along the chamber provide additional measurements compared to the standard stainless steel chamber that has 1 SIP and 1 NEG pump there.

CELL6 : CV3 "SOLEIL" PROFILE NEG COATED NEW CV4

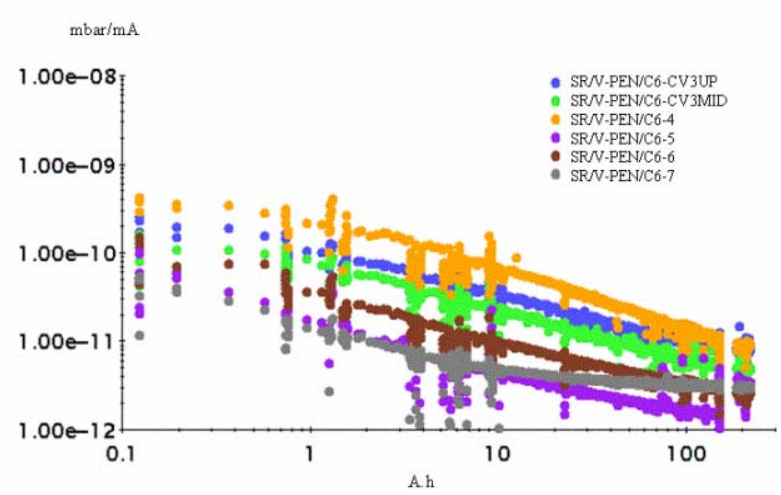

Figure 1: Conditioning of ESRF storage ring cello6 in January 2005 with NEG-coated quadrupole chamber

Figure 1 shows the pressure readings normalised to the machine current versus the integrated beam dose. After restart of the accelerator, the gauge at the exit of the coated chamber (C6-4) started lower and conditioned quicker than its counterpart in another cell that had been vented and reconditioned for comparison (not shown). The gauges along the chamber (C6-CV3UP and C6CV3MID) were even lower by more than a factor of two, despite the fact that they are not located like all the other gauges in front of a sputter-ion pump. No abnormal increase of bremsstrahlung was observed. The gauges labelled C6-5, C6-6, C6-7 are located at the crotch 1, before dipole 2 , and at crotch 2 , respectively, further downstream of the test CV3. They are installed on chambers which had not been replaced during the machine shutdown, but simply vented and re-baked at a low temperature (80C for $>48$ hours). In our experience, chambers which undergo a venting followed by lowtemperature baking still retain some of the photonconditioning previously done by the intense beams of the ESRF.

\section{Small Conductance Cross-Section}

In order to increase the photon beam brilliance of future light sources, the vertical emittance is made as small as possible. This dictates the use of small cross section magnets which, in turn, lead to small cross-section and conductance limited vacuum chambers.

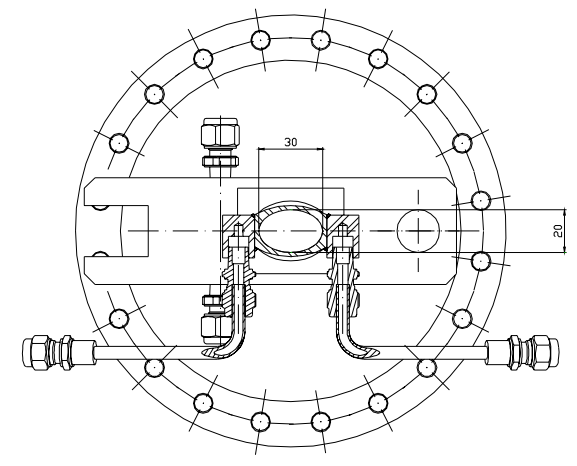

Figure 2: Cross section of experimental ESRF quadrupole chamber.

The ESRF has produced a chamber with an elliptical cross section beam chamber of $30 \mathrm{~mm}$ by $20 \mathrm{~mm}$. It is going to be coated at the ESRF, and will replace the "SOLEIL-type" extrusion discussed above in June 2005 for test. In-situ baking equipment has been provided by CERN as developed for the warm sections of LHC. This chamber will further confirm or deny the feasibility of a pump-less solution.

\section{NEG COATING FACILITY}

\section{$I^{\text {st }}$ Tool Status}

The coating tool had been designed taking into account considerations and advice from CERN in the frame of a license agreement [4]. It had been optimized according to the very stringent constraints of coating with three cathodes the $8 \mathrm{~mm}$ by $57 \mathrm{~mm}$ beam chamber of ESRF insertion device vacuum chambers. A PC-controlled full acquisition of all process parameters has been realised, the stability of the sputtering current is controlled by means of the external field strength and the DC solenoid will be moved automatically to a new position once the coating in another has been finished.

\section{New Coating Tool}

Compared to the actual 1 metre solenoid set-up, the new coating tool will deliver higher magnetic fields (up to 900 Gauss, as compared to the actual 550 Gauss on the $1^{\text {st }}$ tool ), especially for the initial phase of the sputtering. It will also provide the possibility to increase the active length of the magnetic field ( $\left.\mathrm{B}>80 \% \mathrm{~B}_{\mathrm{MAX}}\right)$ by adding a $2^{\text {nd }}$ DC solenoid by more than a factor of two, therefore shortening the coating duration.

The possibility to move by means of motorized translation the upper fixation point for the vacuum chamber that needs to be put vertical for the coating procedure allows to adapt for vacuum chambers between three and five meter in length. The variable magnet sledge for the fixation of the solenoids is designed to receive alternatively a permanent magnet device replacing the DC coils: The permanent magnet solution has advantages for 
chambers which do not fit into the "bore" of the solenoid because of their geometry, the presence of pumping ports or fixed cooling tubes and has been tested at ESRF as well.

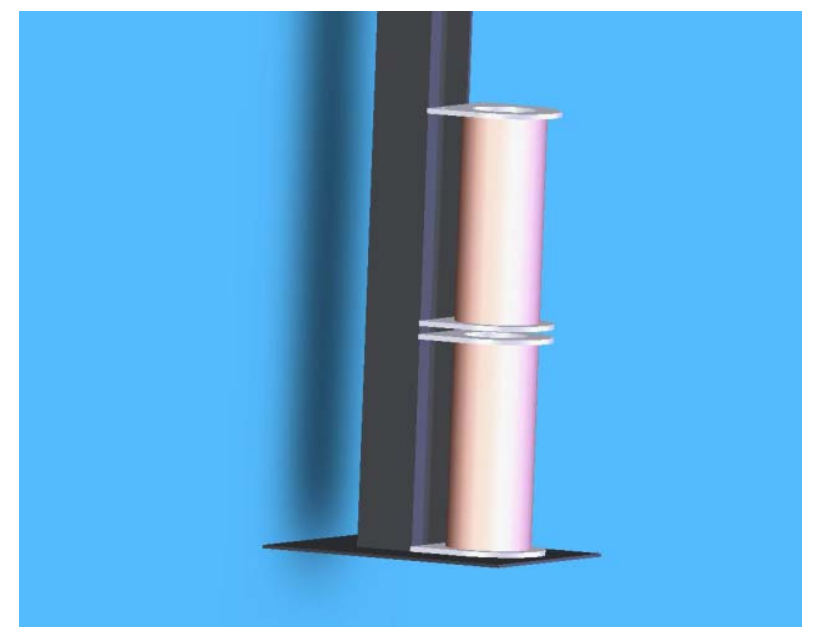

Figure 3: Double solenoid magnet for new coating tool.

\section{CONCLUSIONS}

The NEG coating technology is the state of the art for low gap, out-of-vacuum, insertion device vacuum chambers. It allows the use of aluminium, a material which normally displays high SR-induced desorption yields, thus benefiting from aluminium's better thermal properties and electrical conductivity as compared to stainless steel, avoiding the need for copper coatings. The ESRF has a vast experience with a large number of outof-vacuum ID chambers coated with NEG. Some of them have been installed for several years, without any need for re-activation of the NEG film.

A first test has demonstrated that, deposited on quadrupole chambers, the NEG coating can replace traditional lumped pumping. A future, more stringent test is planned in this direction: if positive, it will demonstrate that "pump-less" solutions are a viable alternative to the standard design of the vacuum system for future highbrilliance SR storage rings.

A second NEG-coating system is being readied for commissioning. It will allow the production of more chambers for the ESRF and for the many laboratories which have expressed interest into this exciting and promising technology and are now moving into its utilization.

\section{ACKNOWLEDGEMENTS}

Many thanks to the Vacuum Group, in particular to I. Parat, D. Schmied, F. Bodart, F. Demarcq and V. Marion. The continuous support of the actual ESRF Machine Director P. Elleaume as well as his predecessor JM Filhol is highly appreciated. Thanks also to the head of the ESRF Technical Service division (P. Thiry), the Safety Group (P. Berkvens) and B. Plan, T. Guenzel, P. Duru from ESRF. We also acknowledge the fruitful collaborations and interesting discussions with our colleagues from CERN, especially P. Strubin, P. Chiggiato and C. Rathjen.

\section{REFERENCES}

[1] R. Kersevan, Proceedings of EPAC 1998, http://accelconf.web.cern.ch/AccelConf/e98/PAPER S/TUP03C.pdf

[2] R. Kersevan, Proceedings of EPAC 2000, http://accelconf.web.cern.ch/AccelConf/e00/PAPER S/THP5B11.pdf

[3] R. Kersevan, Proceedings of EPAC 2002, http://accelconf.web.cern.ch/AccelConf/e02/PAPER S/WEPDO029.pdf

[4] C. Benvenuti, P. Chiggiato, private communication

[5] T.F. Guenzel, T. Perron, L. Farvacque, JL. Revol, "Evolution of the machine impedance following the ESRF upgrade to low gap NEG coated aluminium chambers", this conference.

[6] P. Chiggiato, R. Kersevan, Vacuum 60, 2001, p.6772

[7] Level JP, Filhol JM et al, Proceedings of PAC 2003, http://accelconf.web.cern.ch/AccelConf/p03/PAPER S/TOPA003.PDF

[8] P. Chiggiato, European Vacuum Conference 2004 Venice, to be published in Vacuum

[9] N.B. Mistry et al., "Massive Titanium-Sublimation Pumping in the CESR Interaction Region", PAC97, http://accelconf.web.cern.ch/accelconf/pac97/papers/ $\mathrm{pdf} / 8 \mathrm{C} 006 . \mathrm{PDF}$

[10] V. V. Anashin et al., "Comparative study of photodesorption from TiZrV coated and uncoated stainless steel vacuum chambers", Vacuum 75, 2004, p. $155-159$

[11] R. Kersevan, "Analysis of a Calibrated-Leak Experiment on a Narrow-Gap Vacuum Chamber", poster presented as a post-deadline contribution to the PAC2003 conference, http://www.esrf.fr/Accelerators/Reports/PAC03/kerse van_pac_03 